\title{
SURGE RECOVERY TECHNIQUES FOR THE TEVATRON COLD COMPRESSORS
}

\author{
A. Martinez, A. L. Klebaner, J. N. Makara, and J. C. Theilacker \\ Fermi National Accelerator Laboratory \\ Batavia, Illinois, 60510, USA
}

\begin{abstract}
The Fermilab Tevatron cryogenic system utilizes high-speed centrifugal cold compressors, made by Ishikawajima-Harima Heavy Industries Co. Ltd. (IHI), for highenergy operations [1]. The compressor is designed to pump $60 \mathrm{~g} / \mathrm{s}$ of $3.6 \mathrm{~K}$ saturated helium vapor at a pressure ratio of 2.8, with an off-design range of 40 to $70 \mathrm{~g} / \mathrm{s}$ and operating speeds between 40 and $95 \mathrm{krpm}$. Since initial commissioning in 1993, Tevatron transient conditions such as quench recovery have led to multiple-location machine trips as a result of the cold compressors entering the surge regime. Historically, compressors operating at lower inlet pressures and higher speeds have been especially susceptible to these machine trips and it was not uncommon to have multiple compressor trips during large multiple-house quenches. In order to cope with these events and limit accelerator down time, surge recovery techniques have been implemented in an attempt to prevent the compressors from tripping once the machine entered this surge regime. This paper discusses the different methods of surge recovery that have been employed. Data from tests performed at the Cryogenic Test Facility at Fermilab as well as actual Tevatron operational data were utilized. In order to aid in the determination of the surge region, a full mapping study was undertaken to characterize the entire pressure field of the cold compressor. These techniques were then implemented and tested at several locations in the Tevatron with some success.
\end{abstract}

KEYWORDS: Cold Compressors, Surge, Centrifugal, Test Facilities, Tevatron PACS: 07.20.Mc

\section{INTRODUCTION}

The Tevatron superconducting magnets are cooled by a hybrid cryogenic system that consists of a Central Helium Liquefier (CHL) and 24 satellite refrigerators. Each of the satellite refrigerators cools two 125 m magnet strings. A single stage cold compressor is used in each of the 24 satellite refrigerators to reduce the pressure of the two-phase helium 


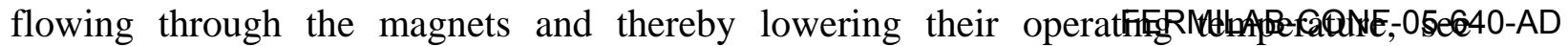
FIGURE 1.

The primary machine is a high-speed centrifugal compressor supplied by IHI. This turbo machine is driven by a small $1.25 \mathrm{~kW}(1.68 \mathrm{hp}), 208 \mathrm{~V}$, 3-phase induction motor powered by a Toshiba inverter controller. The vertical rotor assembly consists of a steel shaft and squirrel cage type rotor with a shroudless, cast aluminum impeller. The assembly is supported radially by two identical self-acting foil gas bearings and axially by a spiral groove gas thrust bearing.

The cold compressors were installed and commissioned in 1993 as part of a low temperature upgrade but were not used to increase the Tevatron's energy until the 2000 collider run [1,2]. Since then, the cold compressors have been running continuously as an integral part of the cryogenic system and collider complex. Cold compressor operating experience in the Tevatron over the last 6 years has evolved considerably. One of the main issues affecting cold compressor operation occurs during accelerator magnet quenches.

A magnet quench occurs when a portion of the magnet's superconductor goes to its normal resistive state. In order to protect the magnet's superconductor from overheating, the quench protection system fires heaters in the magnets to spread out the absorption of the stored electrical energy. It also turns off electrical power supplies, actively dumps magnet energy, initiates quench bypass circuits and communicates quench events to accelerator and refrigerator control systems. Each magnet is protected from overpressurizing by a quench relief valve. This relief valve is mounted external to the magnet and vents to the refrigerator suction which in turn is vented to atmosphere through multiple parallel plate relief valves. This sudden increase in pressure is seen at the discharge end of the cold compressor.

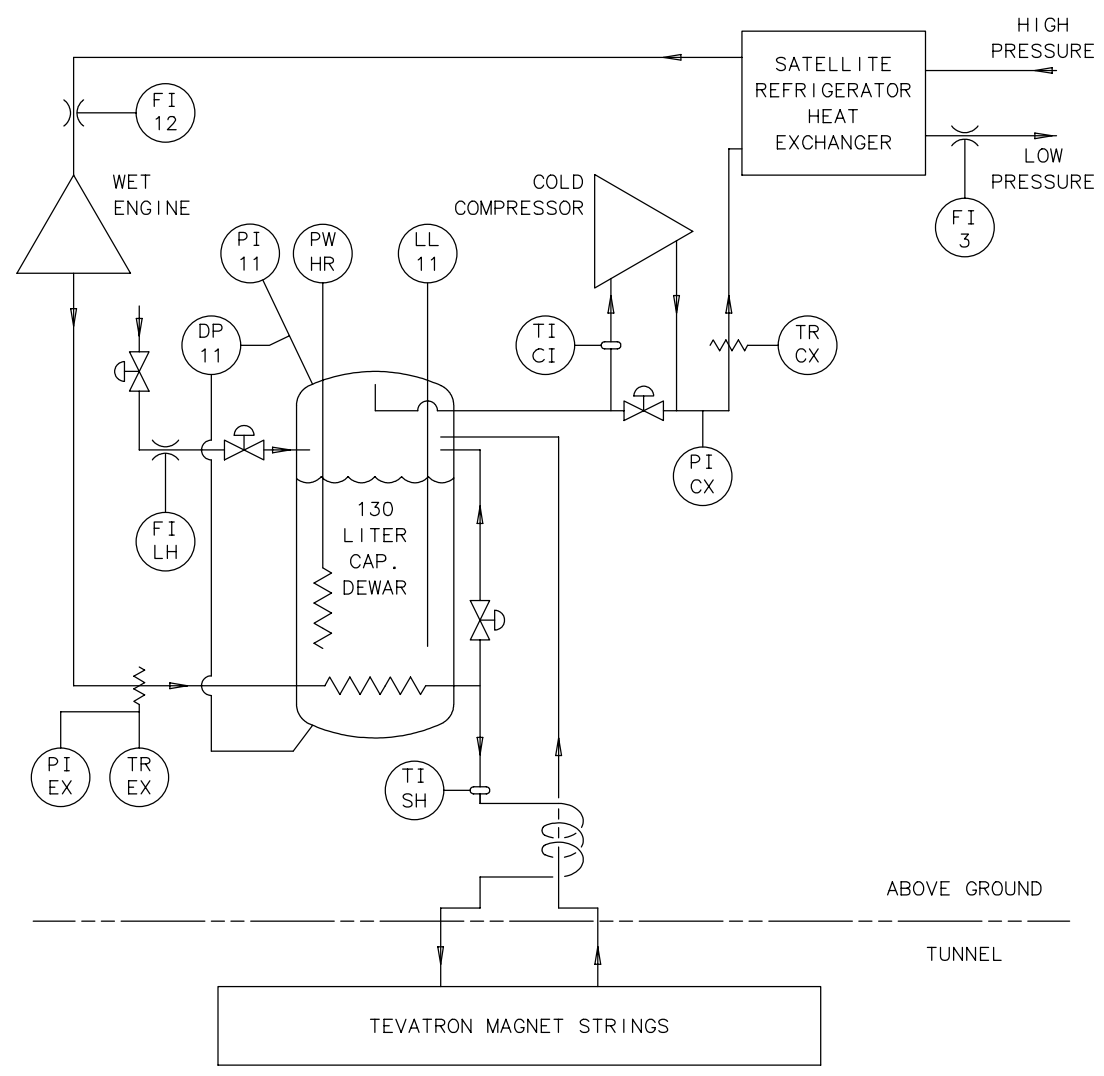

FIGURE 1. Satellite Refrigerator with Cold Compressor. 


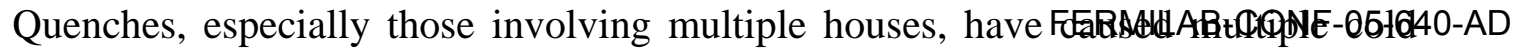
compressor trips. On some large high energy quenches, as many as eight cold compressors have tripped off at a given time. These trips contributed to extended accelerator downtime and more wear and tear on the machines themselves from multiple start and stop cycles. Typically, cold compressors operating at lower inlet pressures and higher speeds have been especially susceptible to these transient effects. A thorough analysis of these events determined that these trips were caused by the cold compressors going into surge and subsequently tripping on motor overload upon recovering from the surge event. Therefore, tests were performed at the Cryogenic Test Facility to completely map the cold compressor pressure field to better understand the surge characteristics of the compressor. These results allowed for better tuning and system behavior under steady state and upset conditions to avoid cold compressor operation near the surge and choke regions. Using this test data coupled with Tevatron operational data, a surge recovery strategy was devised, implemented and tested.

\section{COLD COMPRESSOR SURGE}

In September of 2004, cold compressor tests were performed at the Cryogenic Test Facility at Fermilab to map the hydrodynamic pressure field of the IHI cold compressor, see FIGURE 2. The map is presented in traditional dimensionless coordinates, pressure ratio versus reduced mass flow rate $[3,4]$. The pressure ratio is the ratio of the outlet pressure to the inlet pressure, while reduced mass flow is the actual mass flow through the compressor scaled by the inlet conditions and referenced to the design point parameters [3]. These tests were a continuation of the tests performed the previous year and presented at the Cryogenic Engineering Conference in 2003 [5].

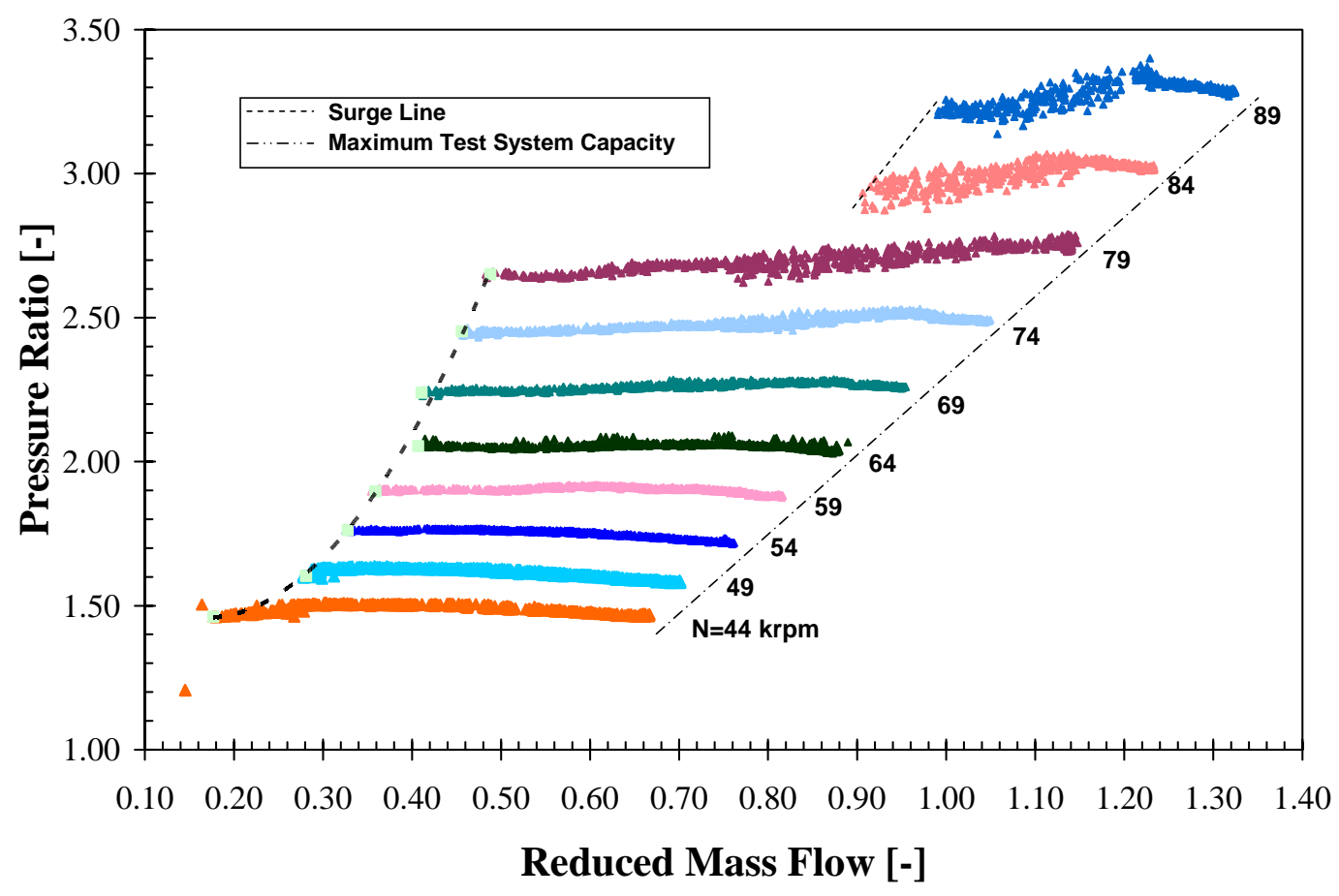

FIGURE 2. Tevatron Cold Compressor Hydrodynamic Pressure Field. 


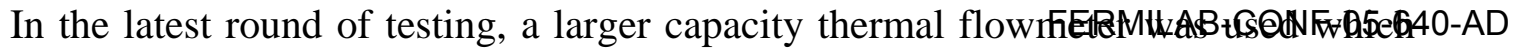
allowed for a greater range in flow rates. Unfortunately, limited by the refrigeration capacity of the system, we were unable to reach the choke line, the line characterized by sonic velocity in some section of the machine where further increase in flow is not possible. Therefore the far right portion of the hydrodynamic map is an artificial end point.

The far left portion of the hydrodynamic map is called the surge point. As the mass flow rate through the cold compressor is reduced, an eventual breakdown of stable flow conditions occur and surge is encountered. This is a condition of turbulent instability in which mass flow fluctuates in and out of the compressor and pressure ratio oscillates sharply. The surge event occurs when forward flow through the compressor can no longer be maintained, due to an increase in pressure across the compressor causing a momentary flow reversal. This flow reversal eventually reduces the discharge pressure and increases the suction pressure, thus allowing forward flow to resume again until the pressure rise again reaches the surge point. This surge cycle will continue until a change is made in the process or compressor conditions. Tests at the Cryogenic Test Facility show that the surge cycle is approximately 45 seconds in length and continues indefinitely unless a process change is made to take the machine out of the surge region or the compressor trips off on motor overload, see FIGURE 3. Although the leftmost point on the hydrodynamic map is the surge point, the region of negative slope in each constant speed line leading up to this point is called the weak or mild surge region. Operation in this region is more sensitive to process changes. This sensitivity makes the cold compressor more susceptible to surging.

In the Tevatron, during a quench event, typically a cold compressor undergoes only one surge cycle. This is because a quench is a highly transient event with peak pressures being reached within $280 \mathrm{~ms}$ of quench onset [6]. The momentary increase in discharge pressure of the cold compressor rapidly reduces the flow rate of the cold compressor and increases the pressure ratio, driving the compressor towards the surge region. As the quench pressure quickly dissipates, the process conditions return to normal and the compressor exits the surge region.

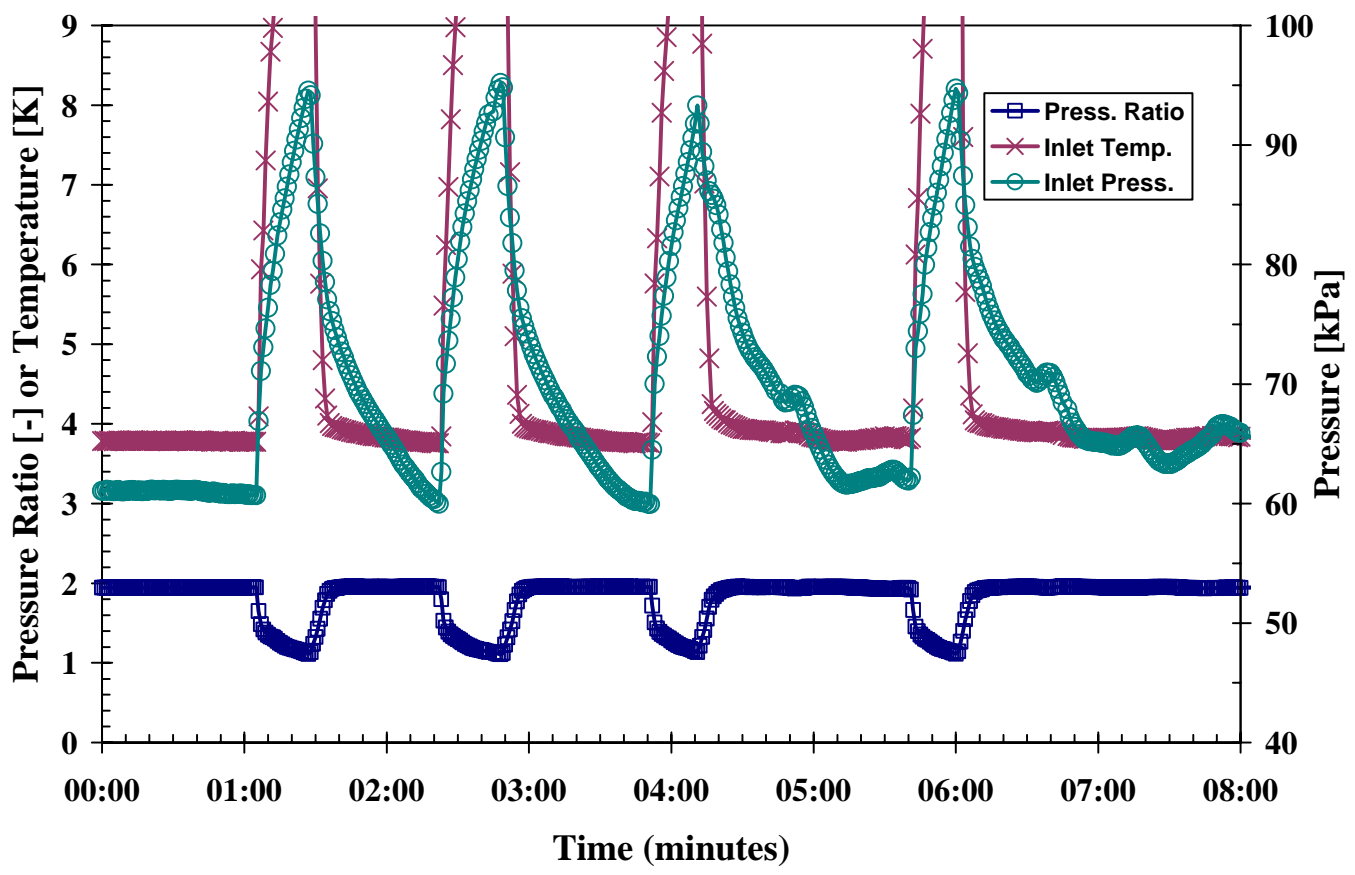

FIGURE 3. Cold Compressor Surge Cycle at CTF. 


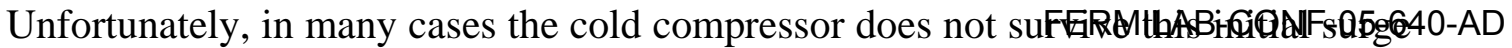
event and trips on high motor overload. During the first stage of the surge cycle, forward flow through the compressor is no longer maintained. This drives the pressure ratio down to 1.0 as compressor suction and discharge equalize. This has the effect of unloading the compressor. When forward flow resumes, a large load is put on the motor as the impeller engages. It is at this point where the cold compressor is susceptible to motor overload trips.

\section{SURGE RECOVERY TECHNIQUES}

The surge recovery scheme was automated using sequential cryogenic control algorithms, called finite state machines (FSM's). The FSM software is the highest level of software in the cryogenic control system [7]. It is used to automate such tasks as quench recovery, cooldown, cold compressor pumpdown, power lead control, etc.. The FSM's work in conjunction with the control system I/O such as PID loops, analog inputs and digital outputs to monitor process conditions and responds accordingly. Operator interface software allows for changing of FSM software parameters and algorithms as well as monitoring of various FSM activities via graphical displays.

Each of the 24 Tevatron refrigerators has a dedicated FSM for cold compressor operation. The FSM is a continuously running program with several features, see FIGURE 4. First, if a quench is detected at a particular refrigerator house, the respective cold compressor at that location is automatically turned off to aid in quench recovery and to protect the machine from possible two phase helium intake. Second if a quench is detected elsewhere in the Tevatron, each non-quenched refrigerator will initially lock the cold compressor speed. This will prevent the cold compressor from ramping up in speed in response to the quickly rising system suction pressures resulting from the quench. In this mode the cold compressor speed will remain locked until pressures drop back to within normal levels. Although in many cases, the cold compressor does not go into surge during this event, we have found that by locking the speed we can reduce the load on the motor and prevent overload conditions. Third, if during a quench a cold compressor enters a surge, as detected by rapidly increasing inlet temperature, the cold compressor speed will be reduced by a defined amount, currently $10 \mathrm{krpm}$, and locked until pressures return to within normal levels. Dropping the speed reduces the load imposed on the motor when forward flow resumes and the compressor begins pumping again. We speculate that this is the reason why cold compressors running at lower pressures and higher speeds have been more susceptible to overload trips.

Development of a proper cold compressor surge response algorithm occurred during normal accelerator operations, taking advantage of available data gained from numerous quenches. Originally, cold compressor speed reductions of 15 and $20 \mathrm{krpm}$ were tested. Although these larger steps were successful in taking the cold compressor out of the surge regime, the larger drop in speed translated to increased compressor inlet pressure and temperature eventually causing excess liquid inventory from the magnet strings to build in the dewar. This resulted in the dewar overfilling with the possibility of two phase liquid intake in the cold compressor. The dewar level can be reduced using a heater but we have found that at the higher wattages required, the vapor produced would rapidly increase the mass flow through the compressor and possibly overload the compressor once it is allowed to regulate. We have found that the $10 \mathrm{krpm}$ step change is sufficient to take the cold compressor out of surge while minimizing dewar overfill effects and recovery. 


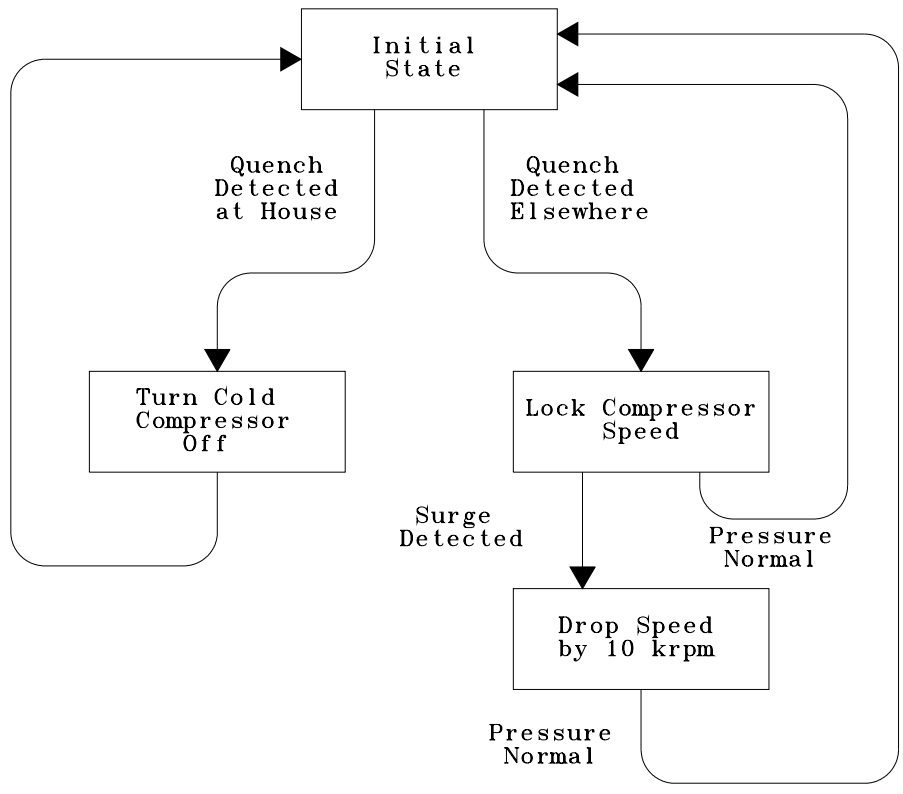

FIGURE 4. Cold Compressor Surge Recovery Algorithm.

\section{TEVATRON OPERATIONAL DATA}

FIGURE 5 shows a typical surge recovery after a quench. Once the quench occurs, the cold compressor immediately enters the surge regime as noted by the rapid increase in inlet temperature as well as inlet pressure. Mass flow drops as forward flow through the compressor ceases. Motor amperage also drops as the compressor essentially unloads. Once the surge is detected by the surge recovery FSM, the speed is dropped by $10 \mathrm{krpm}$ and locked until pressures return to nominal levels.

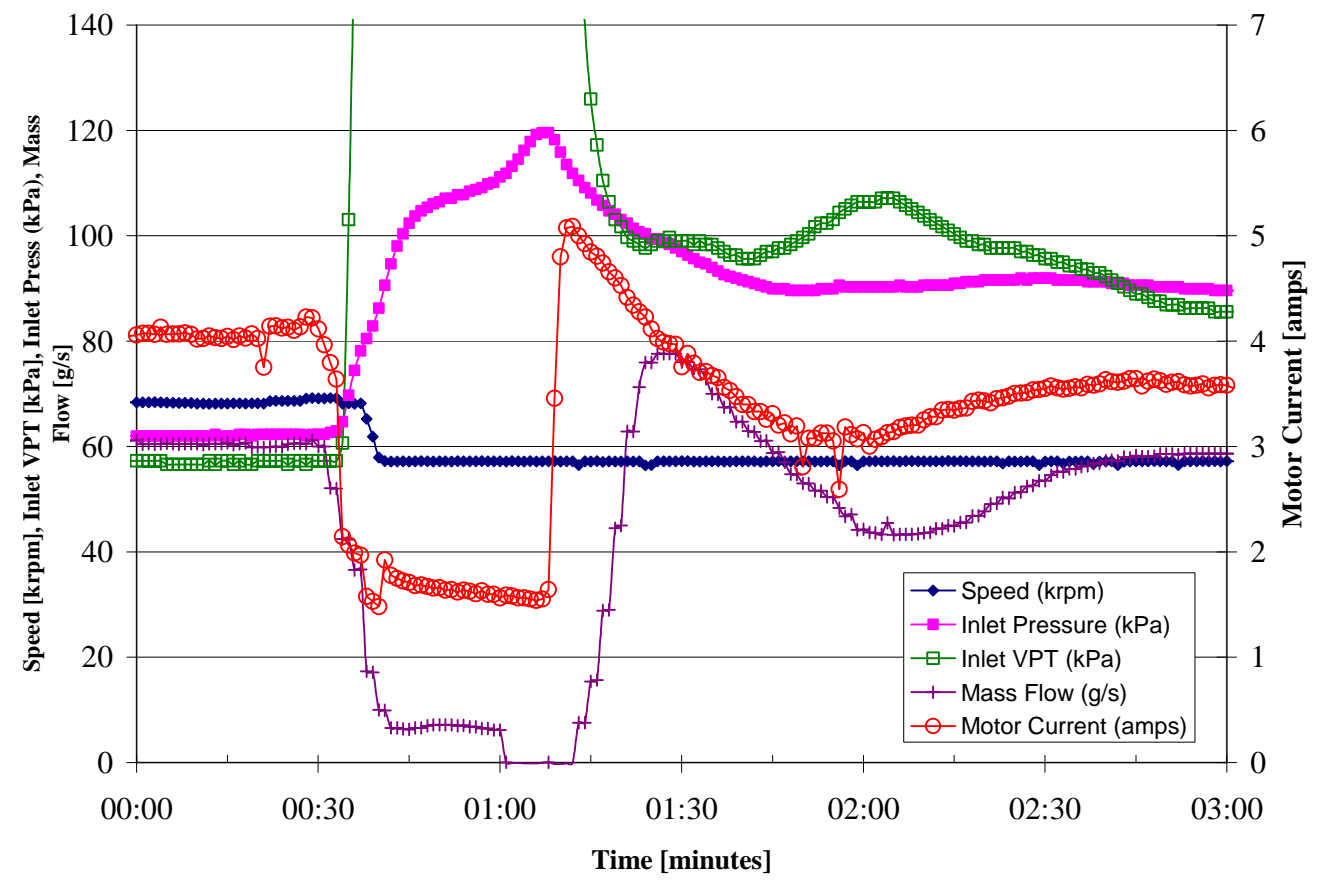

FIGURE 5. Cold Compressor Surge with Automatic Recovery. 
TABLE 1. Cold Compressor Quench Data for July 2005 and July 2004.

\begin{tabular}{cccc|lccc}
\hline Date & $\begin{array}{c}\text { Houses } \\
\text { Quenched }\end{array}$ & $\begin{array}{c}\text { Cold Compr. Cold Compr. } \\
\text { Surges }\end{array}$ & $\begin{array}{c}\text { Date } \\
\text { Trips }\end{array}$ & & $\begin{array}{c}\text { Houses } \\
\text { Quenched }\end{array}$ & $\begin{array}{c}\text { Cold Compr. Cold Compr. } \\
\text { Surges }\end{array}$ & \begin{tabular}{c} 
Trips \\
\hline $7 / 14 / 2005$
\end{tabular} \\
\hline & 16 & 0 & $7 / 5 / 2004$ & 7 & 15 & 2 \\
$7 / 22 / 2005$ & 1 & 5 & 0 & $7 / 7 / 2004$ & 2 & 3 & 0 \\
$7 / 26 / 2005$ & 3 & 9 & 0 & $7 / 8 / 2004^{*}$ & 7 & 17 & 0 \\
$7 / 28 / 2005$ & 7 & 15 & 0 & $7 / 20 / 2004$ & 1 & 2 & 0 \\
$7 / 30 / 2005$ & 7 & 14 & 0 & $7 / 23 / 2004$ & 2 & 3 & 0 \\
& & & & $7 / 30 / 2004^{*}$ & 7 & 12 & 1 \\
& & & & $7 / 31 / 2004$ & 7 & 14 & 5 \\
\hline
\end{tabular}

* Multiple Quenches on these dates

The surge response algorithm has been shown to drastically reduce trips of centrifugal cold compressors due to surges resulting from nearby quenches. TABLE 1 shows a recent month, though not a typical one, where 5 high-energy quenches totaling 24 refrigerator houses resulted in 59 cold compressor surges without any trips upon recovery. This compares well to data from a year earlier, July 2004, where 10 quench events among 33 houses caused 66 cold compressor surges that resulted in 8 cold compressor trips on overload.

The hydrodynamic pressure field of the centrifugal cold compressors provides an invaluable tool for system tuning. Operators can actively tune magnet flows using online software displaying the cold compressor operation relative to the defined surge lines. This tuning also has the benefit of reducing the cold compressor speeds thus reducing the refrigeration load on the system overall. FIGURE 6 shows a recent steady state operational plot of all twenty IHI cold compressors superimposed on the previously established hydrodynamic pressure field map. The remaining four cold compressors are of a reciprocating type and therefore not shown.

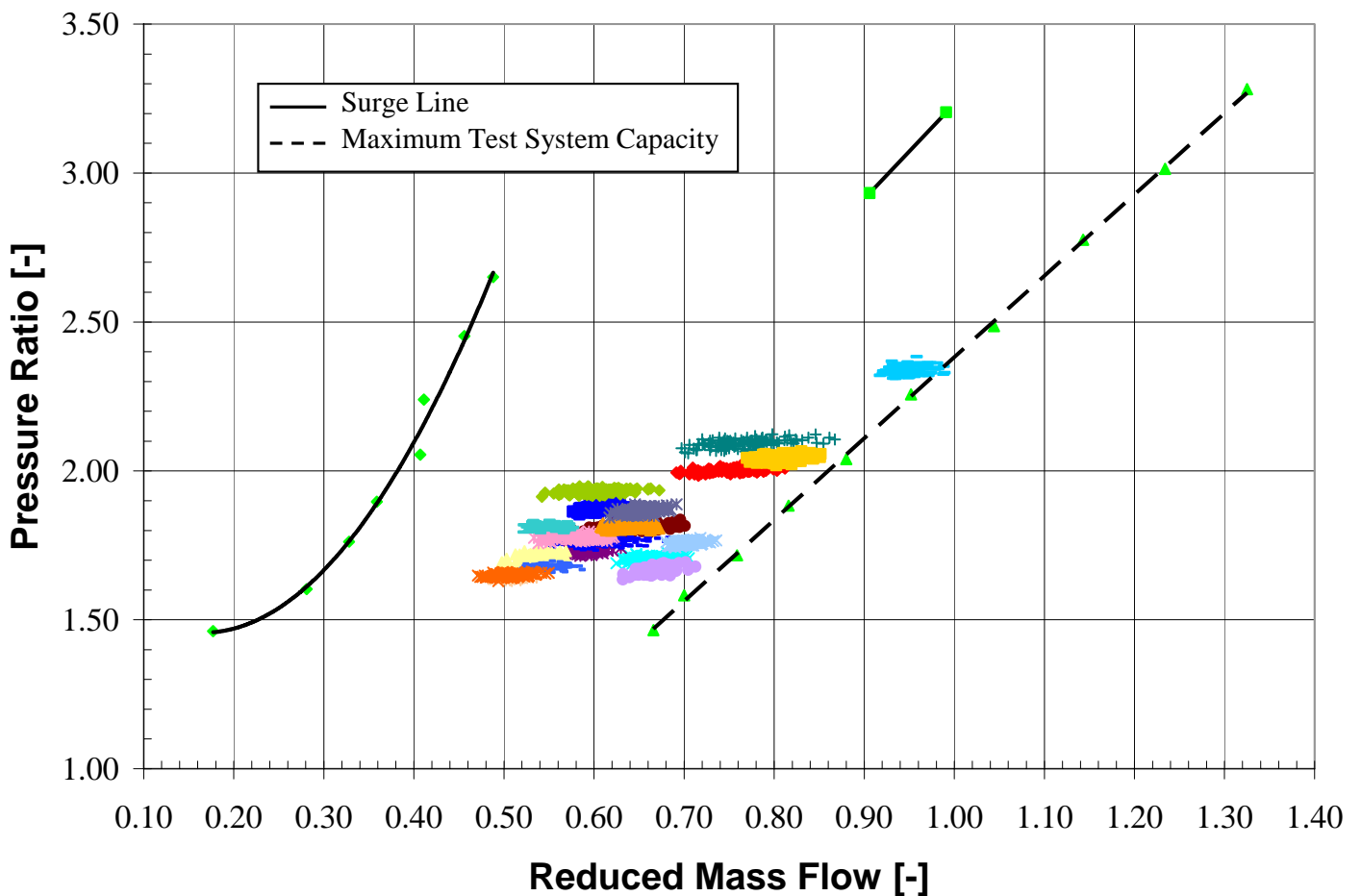

FIGURE 6. Tevatron Cold Compressor Operational Hydrodynamic Pressure Field (all 20 refrigerators). 


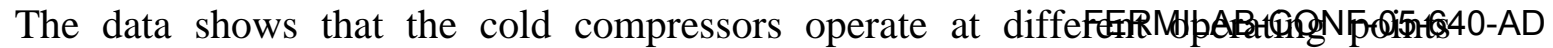
depending on the different heat loads and magnet quench performance in each individual satellite refrigerator. The steady state operational profile shows that the cold compressors have sufficient margin from the surge line during normal Tevatron operation. Therefore, surging of the cold compressor is typically only a concern during transient events such as a quench.

\section{CONCLUSION}

Tests were performed to map the hydrodynamic parameters and surge points of the Tevatron's centrifugal cold compressors. By understanding where the compressor surge line is located, we were better able to tune each refrigerator to avoid steady state cold compressor operation near the surge region. However, tuning alone does not eliminate the possibility of cold compressor trips due to surge events. The very nature of transient effects such as quenches requires that active and automatic processes be implemented to counter these effects. Because of the rapid increase in pressure during quench events, there is no practical method to prevent a cold compressor from entering the surge regime. However, once a surge is detected, steps can be taken to limit the duration of these effects which if uncontrolled could possibly lead to overload trips and possible component damage. The current surge control strategies employed have virtually eliminated all cold compressor trips due to surging, creating overall operational stability and limiting accelerator downtime.

\section{ACKNOWLEDGMENTS}

The authors are grateful to all those in the Cryogenic and Controls Departments who contributed to these studies. Fermilab is operated by Universities Research Association Inc. under Contract No. DE-AC02-76CH03000 with the U.S. Department of Energy.

\section{REFERENCES}

1. Theilacker, J. C., “Tevatron Cold Compressor Operating Experience,” Cryogenics 34, pp. 107-110 (1994).

2. Theilacker, J. C., “Twenty Years of Tevatron Operation,” in Advances in Cryogenic Engineering 49, American Institute of Physics, Melville, New York, pp. 139-146 (2003).

3. Gistau, G. M., et al., "The $300 \mathrm{~W}-1.75 \mathrm{~K}$ Tore Supra Refrigerator Cold Centrifugal Compressors Report,” in Advances in Cryogenic Engineering 33, Plenum Press, New York, pp. 675-681 (1988).

4. Gistau, G. M., et al., “Application Range of Cryogenic Centrifugal Compressors,” in Advances in Cryogenic Engineering 35, Plenum Press, New York, pp. 1031-1037 (1990).

5. Klebaner, A. L., Martinez, A., Soyars, W. M., Theilacker, J. C., "Pressure Field Study of the Tevatron Cold Compressors,” in Advances in Cryogenic Engineering 49, American Institute of Physics, Melville, New York, pp. 265-271 (2003).

6. Theilacker, J. C., Norris, B. L., Soyars, W. M., “Tevatron Quench Pressure Measurement,” in Advances in Cryogenic Engineering 39, Plenum Press, New York, pp. 469-476 (1993).

7. Norris, B. L., Brubaker, J., Franck, A., Lublinsky, B., Lucas, P., Theilacker, J. C., Seino, K. C., Smolucha, J., “New Cryogenic Controls for the Tevatron Low Temperature Upgrade,” in Advances in Cryogenic Engineering 39, Plenum Press, New York, pp. 1185-1192 (1993). 